\title{
Inflammatory signals and network connections implicate cell-mediated immunity in chronic venous insufficiency
}

\author{
Lena Vodovotz ${ }^{1}$, Ruben Zamora ${ }^{1,2}$, Derek A. Barclay ${ }^{1}$, Yoram Vodovotz ${ }^{1,2}$, Jinling Yin ${ }^{1}$, Julie Bitner ${ }^{1,3}$, \\ John Florida ${ }^{3}$, Efthymios D. Avgerinos ${ }^{1,3}$, Ulka Sachdev ${ }^{1,3}$ \\ ${ }^{1}$ Department of Surgery, University of Pittsburgh Medical Center, Pittsburgh, PA, USA; ${ }^{2}$ Center for Inflammation and Regeneration Modeling, \\ McGowen Institute for Regenerative Modeling, Pittsburgh, PA, USA; ${ }^{3}$ Division of Vascular Surgery, University of Pittsburgh Medical Center, \\ Pittsburgh, PA, USA \\ Contributions: (I) Conception and design: U Sachdev, L Vodovotz, R Zamora, Y Vodovotz; (II) Administrative support: U Sachdev, Y Vodovotz, J \\ Bitner; (III) Provision of study materials or patients: U Sachdev, J Bitner, ED Avgerinos, L Vodovotz, DA Barclay, J Florida; (IV) Collection and \\ assembly of data: L Vodovotz, DA Barclay, J Bitner, Y Vodovotz, J Florida; (V) Data analysis and interpretation: All authors; (VI) Manuscript writing: \\ All authors; (VII) Final approval of manuscript: All authors. \\ Correspondence to: Ulka Sachdev, MD, FACS. Associate Professor of Surgery, Department of Surgery, Division of Vascular Surgery, Site Chief of \\ Vascular Surgery, Magee Women's Hospital, University of Pittsburgh Medical Center, 300 Halket Street Suite 5414, Pittsburgh, PA 15213, USA. \\ Email: Sachdevu2@upmc.edu.
}

\begin{abstract}
Background: The role of inflammation in superficial venous reflux in varicose veins (VVs) is unknown. Computational network modeling has deduced inflammation in experimental and clinical settings. We measured immune mediators in plasma from competent and incompetent leg veins inferring the role of cellular immunity based on cytokine networks.
\end{abstract}

Methods: Temperature was assessed using infrared thermography (IRT) to measure inflammation. Blood was obtained during sclerotherapy or endovenous thermal ablation for VVs. Control subjects underwent phlebotomy from saphenous and forearm veins. Vein segments were harvested during surgery. Demographics, clinical, etiology, anatomy and pathophysiology classification, venous clinical severity scores (VCSSs), and body mass index (BMI) were collected. Twenty-five mediators were measured in serum and vein segments. Means were compared using Mann-Whitney $U$ test. Pearson correlations equaling or exceeding a threshold prompted connections among nodes, and mapped as networks. Spearman correlations were performed between interleukin (IL)-17A and both granulocyte macrophage colony stimulation factor, and IL-10 as indicators of pathogenic and nonpathogenic Th17 cell involvement.

Results: Age, BMI, and VCSSs differed significantly between groups. Temperatures were higher over diseased veins. Plasma concentrations of 20 cytokines differed between control and patient subjects $(\mathrm{P}<0.05)$, and most were lower in patients. C-X-C motif chemokine ligand-9 (aka monokine-induced by gamma interferon), C-X-C motif chemokine ligand 10 (aka IFN $\gamma$ induced protein 10), and soluble IL-2 receptoralpha were higher in patients, but not connected to other mediators in networks. In contrast, IL-17A, IL$12 \mathrm{p} 70$, and interferon gamma were the only mediators that were more highly interconnected in venous insufficiency. IL-17A and granulocyte macrophage colony stimulating factor (GM-CSF) were highly correlated in chronic venous insufficiency (CVI) but not in controls. In tissue, refluxing VVs significantly higher IL-15 expression than competent saphenous veins.

Conclusions: Venous insufficiency associates with age, BMI, skin temperature, and plasma cytokines associated with interferon gamma and possibly IL-17A signaling. The vein wall may be a source of activation of cellular activation, given elevated IL-15 expression. Correlations between IL-17A and GM-CSF suggested a potential role for pathogenic Th17 cells in VVs. Differentially expressed inflammatory networks induced by venous hypertension may reflect or drive venous damage and ulceration.

Keywords: Venous disease; computational modeling; inflammation; T helper cells (Th cells) 
Submitted Feb 09, 2021. Accepted for publication May 17, 2021.

doi: 10.21037/atm-21-688

View this article at: https://dx.doi.org/10.21037/atm-21-688

\section{Introduction}

Chronic venous insufficiency (CVI) is a highly prevalent disease, affecting 25 million adults in the United States (1). Further, it has been estimated that over 2 million adults in the USA (with similar incidences worldwide) have advanced chronic venous disease and experience skin changes $(2,3)$. Venous leg ulcers (VLU) are costly both to patients and the healthcare system, in part due to their high recurrence rate and their psychologically debilitating effects (4-8). While prior venous thrombosis is known to be associated with VLU (9), reflux without a history of DVT can also cause ulcers, and there is currently no method to predict which patients with this type of CVI will eventually develop VLU. It has been shown that endovenous ablation helps promote VLU healing (10). However, preventing ulceration and the need for an invasive procedure is preferable because ulcers may recur even despite ablation (9).

There remains a relative paucity of research on CVI within the cardiovascular literature, despite the high impact of the disease and the millions of people affected by it. While it is known that local inflammation promotes VLU in patients with post-thrombotic syndrome $(11,12)$, less emphasis has been placed on circulating inflammatory changes associated with CVI (13). Therefore, we investigated expression of inflammatory mediators in CVI due to primary venous reflux in order to determine whether targets exist to mitigate disease.

When studying inflammation, computational modeling can provide a holistic look at the entire inflammatory milieu as a function of numerous inflammatory cytokines and chemokines. Our past research utilized dynamic network analysis (DyNA) to generate inflammatory mediator networks based upon plasma levels of cytokines and chemokines collected from an array of disease states, including blunt trauma and pediatric liver failure (14-18). This network inference method was used to infer a role for Th17 cells in both experimental (19) and clinical (17) settings. Furthermore, this analysis was able to effectively separate survivors from non-survivors, since inflammatory network complexity was significantly different for nonsurvivors across all time points (16). Recently, we employed a similar method of network analysis to compare inflammatory mediators between individuals with CVI and banked plasma samples from healthy controls (20). Our current study expands on previous work, including contemporaneously recruited controls, inclusion of thermographic data and analysis of venous tissue (21). We also performed novel analysis to help decipher which immune cells may contribute to disease in CVI. We present the following article in accordance with the MDAR checklist (available at https:// dx.doi.org/10.21037/atm-21-688).

\section{Methods}

\section{Subjects}

\section{Subjects for plasma collection}

The study was conducted in accordance with the Declaration of Helsinki (as revised in 2013). Patients $(n=43)$ and control subjects $(n=8)$ were recruited prospectively and consented for this study under the auspices of IRB protocol \#PRO15030072 issued through the institutional ethics board of the University of Pittsburgh. A minimum of 7 per group had been previously determined to provide enough power to see statistical difference in select mediators based on prior data. Inclusion criteria for patients included: clinical C2 -5 disease of a venous origin, age $\geq 18$ years, ability, and willingness to sign written informed consents for research participation and for treatment, duplex evidence of venous reflux $(\geq 0.5 \mathrm{~s})$ in the superficial venous system, and GSV, AASV, SSV $>5 \mathrm{~mm}$ and/or pathologic varicosities size $>3 \mathrm{~mm}$, guided by clinical criteria for treatment. Exclusion criteria included active or prior deep venous thrombosis (DVT), active VLU, active PAD (ABI $<0.8$; toe pressure $<40 \mathrm{mmHg}$ ), cellulitis, pregnancy, renal insufficiency, myocardial infarction within 6 months, heart failure, connective tissue disease, pneumonia (within 6 months), and immunosuppressive, or anticoagulation use. Patients with clinical, etiology, anatomy and pathophysiology (CEAP) (22) clinical class $\mathrm{C} 2-5$ undergoing either sclerotherapy or endovenous thermal ablation for primary venous insufficiency underwent phlebotomy from either the great saphenous vein (GSV), the anterior accessory saphenous vein (AASV), the small saphenous vein (SSV), or refluxing dilated truncal varicosities (nonsaphenous veins; NSVs) that were targeted 
for treatment as described (20). Samples were collected into ethylenediaminetetraacetic acid (EDTA) for a final volume of $2 \mathrm{cc}$. After centrifugation at high speed for ten minutes, plasma was removed and stored at $-80{ }^{\circ} \mathrm{C}$ until use. Clinical data were collected in a de-identified manner and included age, gender, race, body mass index (BMI), smoking history, data relevant to CEAP classification, and venous clinical severity score (VCSS) (23). CEAP classification data was recorded based on the most recent updates (22) and assessed using both duplex data, physical exam and patient history.

Control subjects were eligible for participation if they denied a history of VVs or telangiectasias that were visible, and did not have significant swelling, aching, fatigue, itching or ulceration at the ankle. Controls subjects underwent phlebotomy from both the saphenous vein near the ankle and the antecubital fossa without the use of a tourniquet to better mimic the conditions in which blood was taken from patients. Samples were collected into EDTA for a final volume of $2 \mathrm{cc}$, and the sample was processed as described above. Control subject age, gender, race, height, weight, smoking history, history of venous treatments or DVT, and VCSS scores were recorded.

\section{Subjects for vein harvest}

Pathologic vein segments were procured from a smaller cohort of patients undergoing phlebectomy for VVs $(n=7)$, or lower extremity bypass $(n=5)$. VV segments were obtained from patients undergoing phlebectomy in the operating room. Control GSV segments were obtained from patients undergoing lower extremity bypass using GSV. Approximately $1-1.5 \mathrm{~cm}$ of vein tissue was harvested and placed directly in RNAlater ${ }^{\circledR}$ tissue storage reagent (Thermo Fisher \#AM7021, Thermo Fisher Scientific, Waltham, MA, USA), and frozen until use.

\section{Infrared thermography (IRT)}

Infrared images were taken of the pathologic veins in a cohort of patients $(n=7)$. For comparison, images were obtained in controls over the GSV by the ankle where the veins are the most superficial and visible beneath the skin and where it potentially experiences the highest hydrostatic pressure similar to VVs ( $n=7)$. Pictures were obtained using a FLIR camera, in a temperature-controlled room at a distance of 1 meter as described, with patients in a standing position (21). The ambient temperature and humidity were consistent across patient and control subjects. The FLIR Tools software (FLIR Instruments, 2009, Wilsonville, OR,
USA) was used for measurement of temperature. Using the oval region of interest (ROI) measurement tool, 3 to 5 measurements of the vein and 3 to 5 measurements from areas remote from the vein were taken. For each ROI, the maximum, minimum, and average temperatures were calculated. Mean temperatures were measured over the vein segments and skin segments remote from the veins. The temperature of fixed elements in the room were recorded as a control. Means temperatures were compared between controls and patients using Students $t$-test.

\section{Sample processing and statistical analysis}

Luminex $^{\mathrm{TM}}$ (EMD Millipore Kit, Billerica, MA, USA) was used to measure the following cytokines and chemokines in duplicate: granulocyte macrophage colony stimulating factor (GM-CSF), interferons alpha2 and gamma (IFN- $\alpha 2$, IFN $\gamma$ ), interleukins (IL)-1 receptor antagonist (IL-1ra), IL1 $\beta$, IL-2, IL-4, IL-5, IL-6, IL-7, IL-8, IL-10, IL-12p40, IL-12p70, IL-13, IL-15, IL-17A, soluble IL-2 receptor alpha chain (sIL-2R $\alpha$ ), tumor necrosis factor alpha (TNF- $\alpha$ ), eotaxin (CCL11), interferon gamma-induced protein 10 (IP-10; CXCL10), monocyte chemoattractant protein (MCP-1; CCL2), monokine induced by gamma interferon (MIG; CXCL9), and macrophage inflammatory protein alpha and beta (MIP-1 $\alpha$, MIP-1 $\beta$; CCR5). This panel was chosen based on their association with other wound healing modeling of similar type $(15,20,24,25)$. Luminex values from plasma were expressed as $\mathrm{pg} / \mathrm{mL}$.

Vein tissue segments were homogenized and protein concentration was assessed using BCA (source). Luminex was performed as described for plasma. Resulting values were expressed as $\mathrm{pg} / \mathrm{mg}$ of tissue.

Mean values of inflammatory mediators, age and VCSS scores and their standard deviations were calculated. Individual inflammatory mediator data was compared between controls and subjects using Mann-Whitney U tests. Network analysis was carried out to define the central inflammatory network nodes in the control and CVI groups using our previously published algorithm. Pearson correlations among mediators (nodes) were performed to determine their dependence on one another. Connections (edges; -) were created if a correlation between two mediators was $\geq$ to a specific threshold Pearson correlation, either an absolute value of 0.7 or 0.95 (equivalent to $\mathrm{P}=0.05)$, as indicated. These cross-correlations were visualized as networks created with MATLAB ${ }^{\circledR}$ software (The MathWorks, Inc., Natick, MA, USA). A robustness 
Table 1 Summary of CEAP classifications of patient subject, and the location of venous sampling

\begin{tabular}{|c|c|c|c|c|c|c|}
\hline \multicolumn{4}{|c|}{ CEAP $[N]$} & \multicolumn{3}{|c|}{ Sampled vein } \\
\hline $2[5]$ & $\mathrm{P}[40]$ & GSVa [20] & & GSV [22] & $2.99 \pm 1.16$ & $7.32 \pm 2.83$ \\
\hline $3[26]$ & $\mathrm{Si}[3]$ & GSVb [2] & Reflux [43] & AASV/SSV [12] & $2.90 \pm 1.38$ & $5.36 \pm 1.08$ \\
\hline $4 a[5]$ & & AASV/SSV [12] & Obstruction [0] & NSV [9] & $2.85 \pm 1.12$ & $6.31 \pm 2.39$ \\
\hline $5[5]$ & & & & & & \\
\hline
\end{tabular}

$\mathrm{N}$ is shown in parenthesis. Mean and standard deviation is shown. Of note, the number of patients shown below is 43 . One sample was not usable. However, all the patients who were sampled are included. CEAP, clinical, etiology, anatomy and pathophysiology; N, number of patients per group; a, above knee, b, below knee; P, primary; Si, secondary; GSV, great saphenous vein; AASV, anterior accessory saphenous vein; SSV, small saphenous vein; NSV, nonsaphenous vein.

index was also calculated to measure network "strength", by comparing the number of connections created with a Pearson correlation of (an absolute value of) 0.7 to one of 0.95 (16). The number of connections for each cytokine was individually calculated for both groups.

Spearman's rank correlation was performed to measure the strength of the association between IL-17A and GM-CSF and between IL-17A and IL-10 (Luminex ${ }^{\mathrm{TM}}$ data) using a modified version of a MATLAB $^{\circledR}$-based toolbox (26) as described previously (17). This rank-based analysis allows for data to be transformed into integer ranks before processing. The algorithm pulls a random permutation sample of the rank input, runs the correlation and repeats the process 1,000 times. The results are applied to a scatter plot of $\mathrm{X}$ rank $v s$. $\mathrm{Y}$ rank, where $\mathrm{X}$ and $\mathrm{Y}$ represent the mediators analyzed. Figures are generated depicting the ranks. A $P$ value of less than 0.05 was considered significant.

\section{Results}

The mean age of the patients ( $\mathrm{n}=43$ sampled) was 54.9 [31-88] years and the mean age of the controls $(n=7)$ was 34.7 [21-47] years, $\mathrm{P}=0.008$. One sample in the patients was not usable. In addition to being older, patients also had a higher BMI $\{30$ [19-48] vs. 23 [20-26], $\mathrm{P}=0.004\}$, and a higher VCSS score (6.5, range, 2-14 vs. 1.1, range, 0-2, $\mathrm{P}<0.001)$. Specific details regarding CEAP classification and the vein that was sampled including type, average reflux time ( $>0.5$ is considered pathologic), and average size are shown in Table 1. Only a minority of patients had secondary insufficiency from obstructive pathology. For thermographic imaging, a total of 7 consecutive patient subjects and 7 consecutive control subjects were recruited for the thermography portion of this study. Infrared imaging demonstrated significant temperature differences between the groups over the course of the vein but not over the remote skin (Figure 1; $\mathrm{P}<0.01$ ). Background temperature did not differ between the control and patient groups.

These findings may reflect an inflammatory process in the diseased veins. To test this hypothesis, we assessed a panel of inflammatory mediators in harvested vein segments from diseased and control veins which were obtained from patient subjects undergoing surgery either for VVs or for lower extremity bypass. These patients were distinct from those undergoing phlebotomy during sclerotherapy or endovenous ablation. Cytokine values in the VV walls demonstrated novel expression patterns. In particular, IL-15 was the only cytokine with significantly higher expression in VVs than the GSV $(\mathrm{P}<0.04$; Figure 2).

We next sought to determine if this local suggestion of inflammation was also reflected in the circulating plasma within the vein. Accordingly, 42 patients and 7 control subject samples were analyzed for circulating inflammatory mediators. To carry out this analysis, we first ascertained that there would be no difference in measured inflammatory mediators based on sampling site. In control subjects, blood was able to be obtained from the leg in 7 people, and from the antecubital fossa without a tourniquet in 5 people. Individual mediator values in plasma obtained from the antecubital fossa did not differ significantly from that in the saphenous vein (Figure 3). Thus, there was little if any variability between sites of phlebotomy in controls. Similarly, there was very little variability of mediator concentrations 


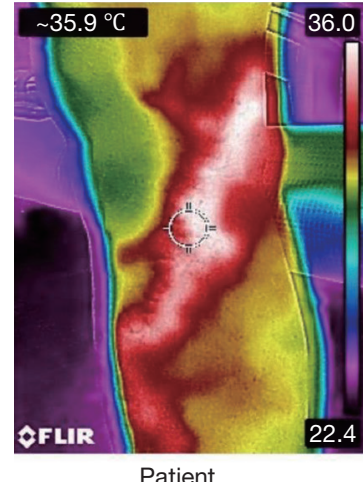

Patient

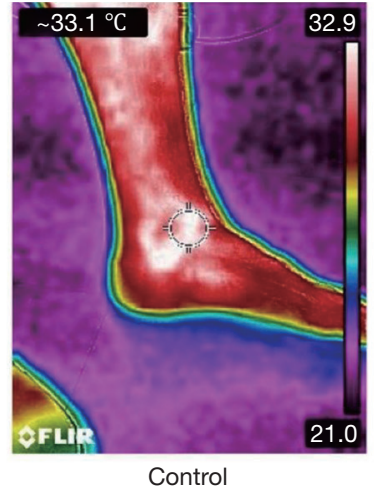

Control

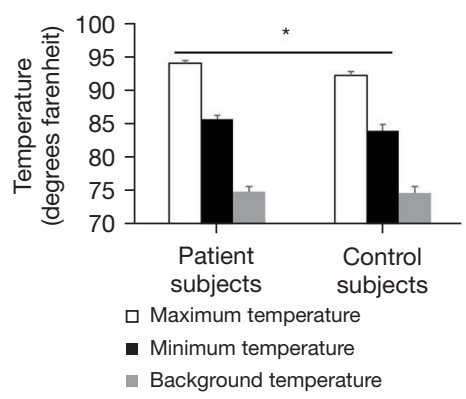

Figure 1 IRT demonstrates skin temperature differences between patient and controls. IRT was performed over the VV targeted for treatment in patients and over the saphenous vein at the ankle for controls. Images reflect heat maps. On the right, corresponding nonthermal images of the veins are shown. Graph depicts mean maximum and minimum temperatures, with error bars representing the standard error of the mean, * $\mathrm{P}<0.01$, patients vs. control, maximum temperature ( $\mathrm{n}=7$ each). IRT, infrared thermography; VV, varicose vein.

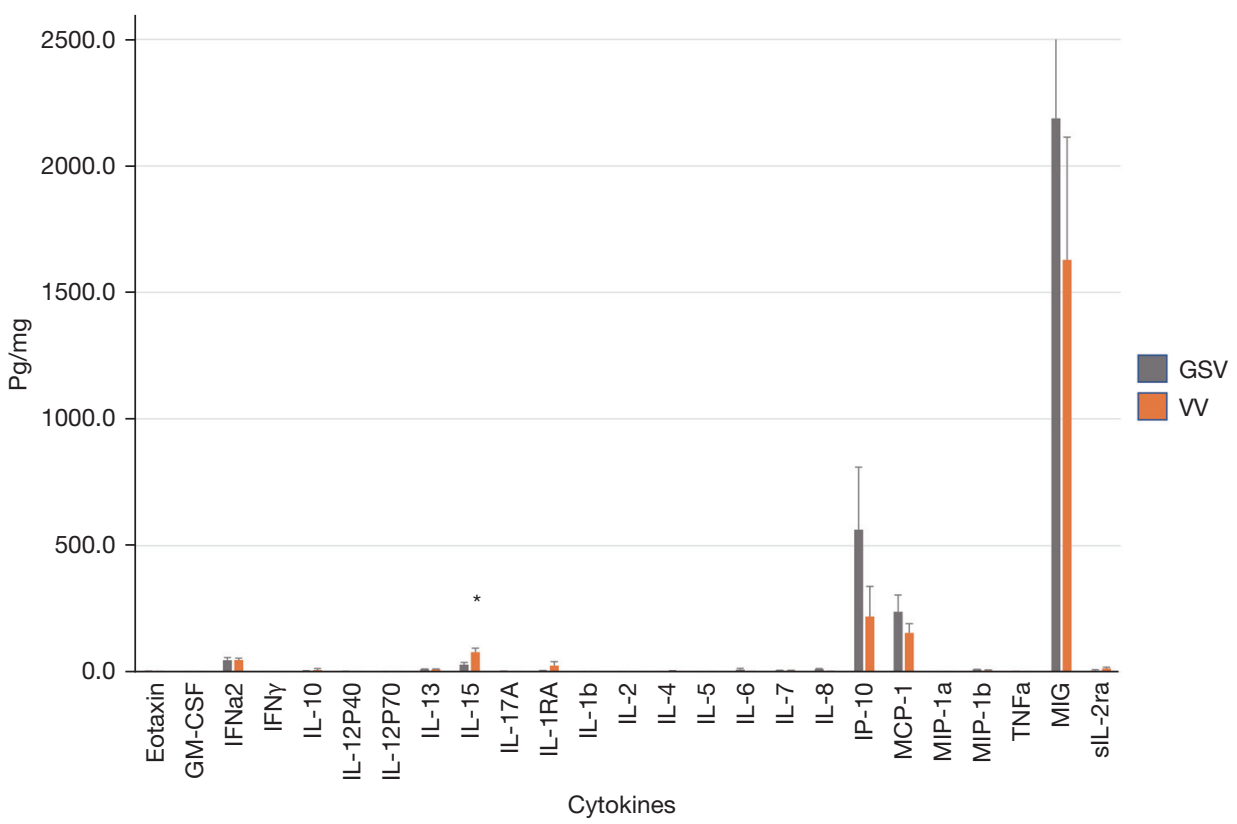

Figure 2 Luminex performed on homogenized vein segments from GSV and VV. Error bars represent standard error of the mean. *, $\mathrm{P}<0.04$ $(\mathrm{n}=5 \mathrm{GSV}, \mathrm{n}=7 \mathrm{VV})$. GSV, great saphenous vein; VV, varicose vein.

among control subjects regardless of the sampling site.

Most individual mediator concentrations in leg vein plasma differed significantly between patient and control subjects. These data are shown in Table 2. Notably, patient subjects had lower concentrations of most inflammatory mediators. However, MIG, IP10, and sIL-2r $\alpha$ were each significantly higher in patients than in controls $(\mathrm{P}<0.001$, $\mathrm{P}<0.001$, and $\mathrm{P}=0.005$, respectively). In the case of $\mathrm{MIG}$, the difference in concentration between patients and controls was delineated further. Patients who were treated only once within the month had higher concentrations than those treated more than one time within a month of the index procedure $[1,362.91 \pm 1,344.77(\mathrm{n}=35)$ vs. $542.66 \pm 219.86$ $(\mathrm{n}=7), \mathrm{P}<0.02, t$-test, mean \pm standard deviation].

We next determined if these differences in circulating inflammatory mediators reflected defined immune/ 


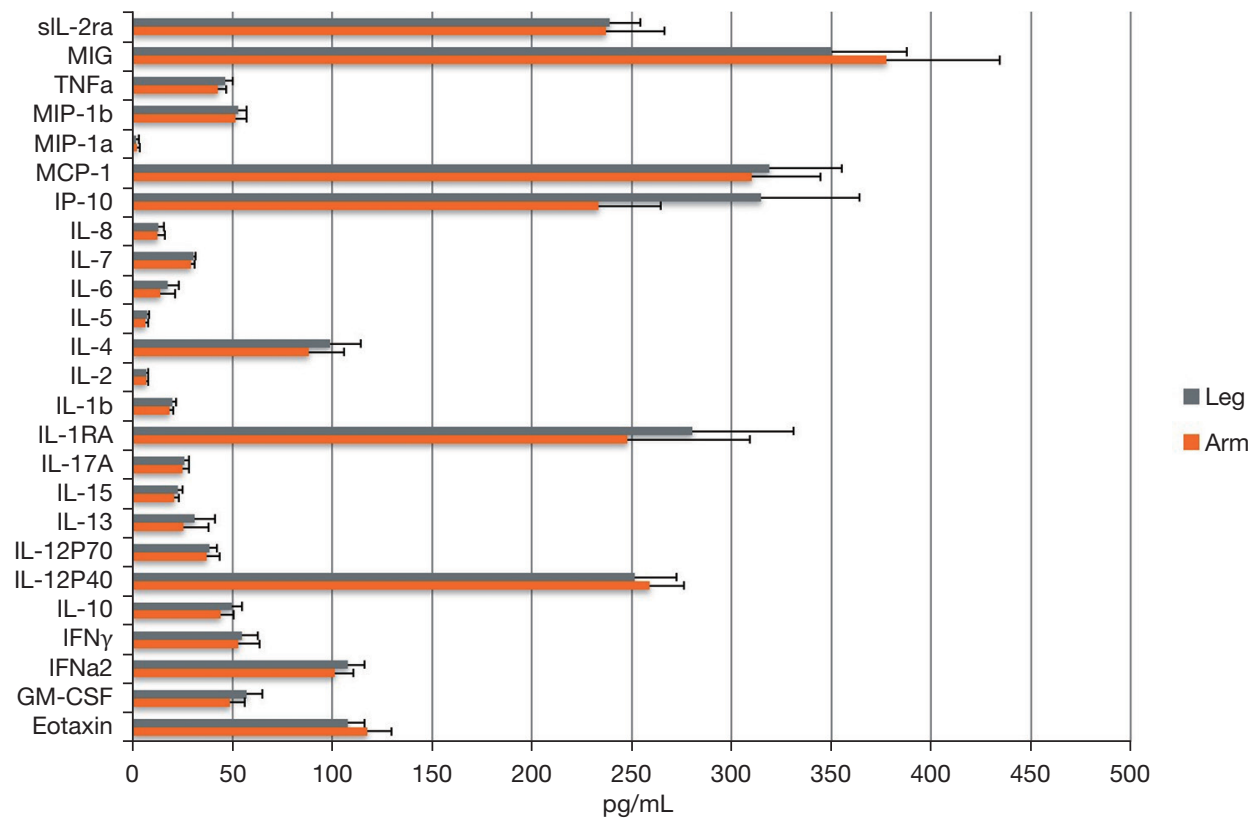

Figure 3 Plasma from the antecubital fossa and saphenous veins show little mediator concentration variability in control subjects. Blood was collected into EDTA from the antecubital fossa and saphenous vein at the ankle in control subjects. A tourniquet was not used in either location. Samples were centrifuged and plasma subjected to multiplex analysis to determine cytokine and chemokine concentrations. Means concentrations and standard error of the mean are shown $(n=7)$. EDTA, ethylenediaminetetraacetic acid.

inflammatory programs. Accordingly, network analyses were performed at a stringency of 0.7 and 0.95 and are shown in Figure 4. The complexity of inflammatory networks was similar between plasma from the arms and legs in control subjects. At stringencies of both 0.7 and 0.95 , network complexity was lower in plasma from patients when compared to plasma obtained from controls at either site. The robustness index, a measure of network strength and hence degree of inflammatory stimulus, was also lower in patients (0.096) vs. controls $(\mathrm{leg}=0.169$ and $\mathrm{arm}=0.241$, respectively).

We then separated the mediators into two groups based on those that were lower in CVI patients (Group I) and those that were elevated in CVI patients (i.e., MIG, IP10 and sIL-2ra; Group II). We evaluated the number of connections that existed among the mediators of these two groups in CVI patients and controls. The results are shown in Table 3. Notably, in group II, where a select group of mediators were higher in CVI, the number of network connections of those mediators was low [Group I: $10.64 \pm 4.81$ vs. $6.64 \pm 3.50$ connections in control vs. CVI $(\mathrm{P}<0.01)$; Group II: $0.67 \pm 0.58$ vs. $0 \pm 0$ connections in control vs. CVI $(\mathrm{P}=0.004)$ ]. Notably, we have previously reported similar findings of cytokines such as IL-6 being "elevated but not connected" in other inflammatory states in which elevated mediators reflect a response to a pathologic insult (14). We also note that systemic elevations in the chemokines MIG and IP10 may be related to the local elevation of IL-15, as has been reported in the context of tumor immunity (27).

Separation of these inflammatory mediators into two groups also highlighted another group of mediators in CVI that were expressed at lower levels than controls but had more connections. These included IFN $\gamma$, IL-12p70, and IL-17A. The data for these cytokines, which were the only three mediators that had more connections in CVI patients than in controls, are depicted graphically in Figure 5.

IFN $\gamma$, IL12p70 (active subunit of IL-12), and IL-17A reflect activation of populations of $\mathrm{T}$ helper (Th) cells that can include both Th1 and Th17 cells $(28,29)$. Indeed, IL12p70 promotes differentiation of CD4+ Th cells into Th1, IFN $\gamma$-producing cells (30). Both cell types have a role in immunity (including autoimmunity). Additionally, Th17 cells can be either pathogenic and nonpathogenic, and can be distinguished based on the co-expression of granulocytemacrophage colony stimulating factor (GM-CSF) or IL-10, 
Table 2 Mediator values between patient and control subjects

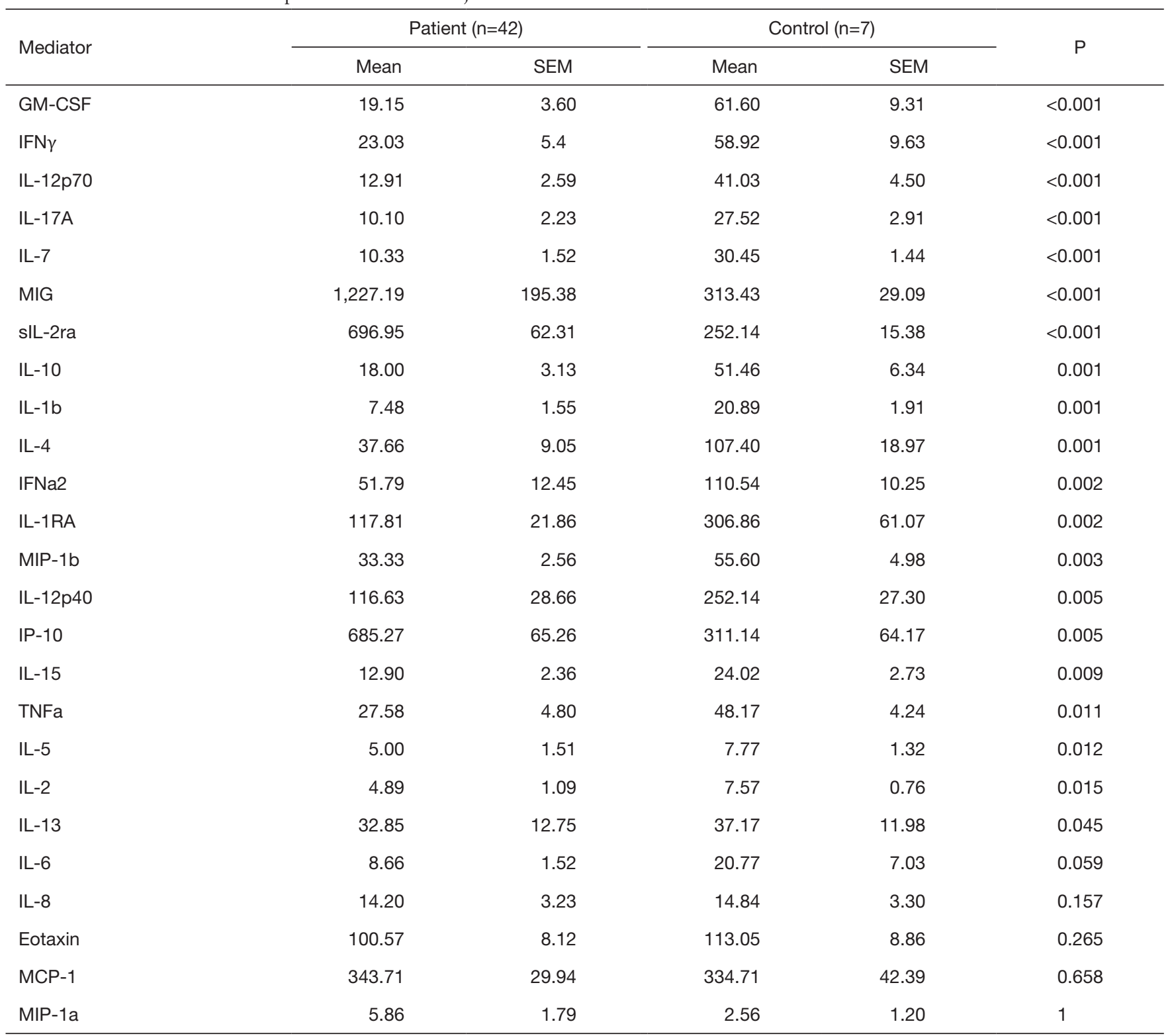

SEM is shown. $P$ values obtained using Mann-Whitney $U$ test. SEM, standard error of the mean; GM-CSF, granulocyte macrophage colony stimulating factor.

respectively $(31,32)$. Because we noticed an increase in the number of IL-17A connections in patient subjects with CVI, we sought to pursue this finding further. We therefore evaluated the potential phenotype of Th17 cells by measuring ranked correlations between IL-17A and GMCSF or IL-10, reflecting pathogenic and nonpathogenic Th17 cells, respectively (16). The results are shown in Figure 6. In CVI patients, IL-17A was tightly correlated with expression of GM-CSF. This correlation was not seen in control patients. In both CVI patients and controls, IL-17A correlated with IL-10 expression. Thus, we infer the presence of pathogenic Th17 cells in CVI patients. Note that the input data are integers and overlap is highly likely. This results in fewer points on the Spearman scatter plot than the number of individual data-points in the raw data. 


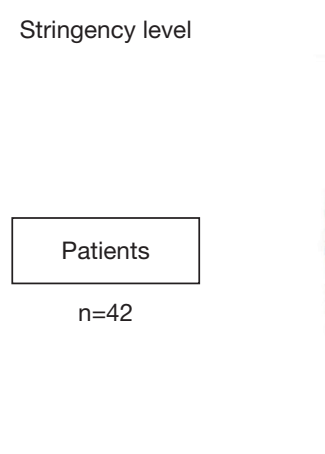

Complexity

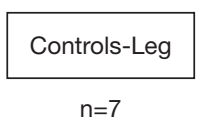

$\mathrm{n}=7$

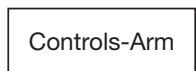

$\mathrm{n}=5$

Complexity

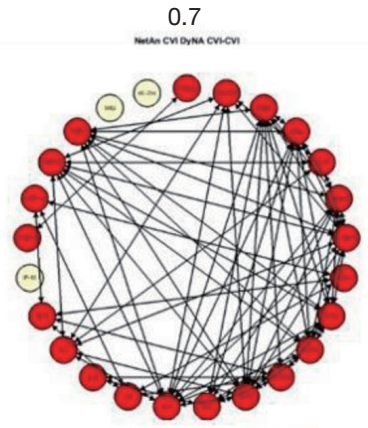

6.0833

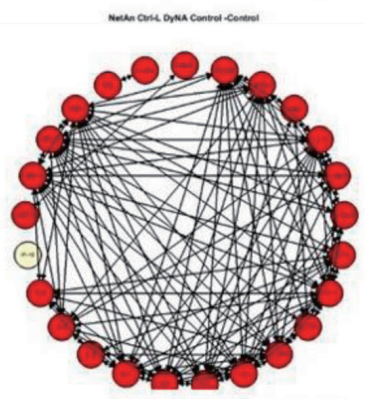

9.8333$$
\text { Complexity }
$$

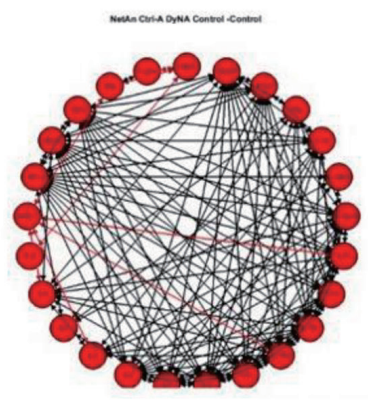

11.0833

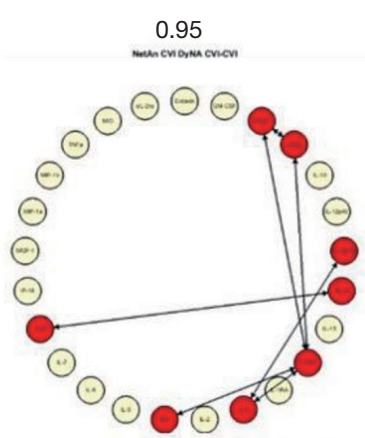

0.5833

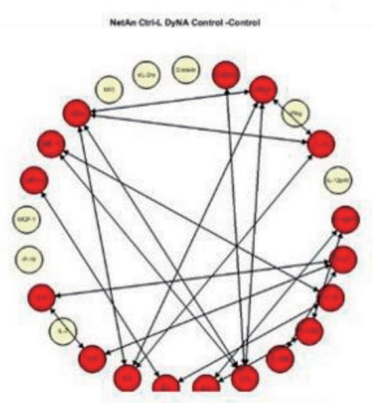

1.6667

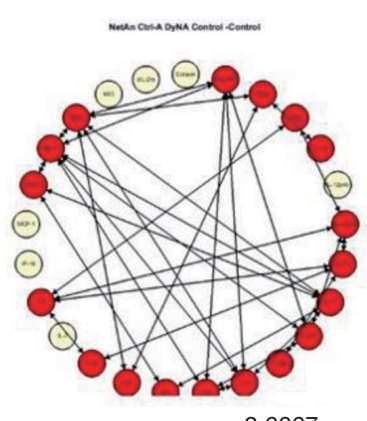

2.6667

Figure 4 Network complexity is diminished in patients when compared to control subjects. Networks of inflammatory mediators were created using MATLAB software as described. Those created at thresholds of 0.7 are shown on the left and those created at 0.95 are shown on the right. Network complexity in both the arm and the leg of control patients is high, suggesting a highly connected and coordinated homeostatic state. Alternatively, the networks in patients were significantly less complex than those of controls at both thresholds. Of note, in the controls, blood could not be obtained from the antecubital fossa in two patients. MATLAB, matrix library.

\section{Discussion}

The inflammatory response to injury occurs in multiple scales, involves the activation of many signaling pathways, may persist for prolonged periods, and may lead to protracted illness. This process is regulated by chemokines and cytokines which interact to form multiple feedback loops. Cytokines are released by cells and affect signaling of other cells. Chemokines are cytokines that initiate movement of cells toward a stimulus. All play critical roles in propagating or attenuating an inflammatory response $(33,34)$. Attenuation of inflammation at a critical time might be expected to correlate with healing, whereas propagation would not. On the contrary, attenuated inflammation from an exhausted immune response may result in disease progression. VVs reflect an abnormal structure of the superficial veins draining the legs, and typically take years to develop (35). We evaluated whether their presence might be a product of a local, chronic inflammatory response in the vein.

Our collaborative team published a prior report 
Table 3 Number of connections/mediators in control vs. CVI patient subjects as determined by Network Analysis (stringency level 0.7)

\begin{tabular}{|c|c|c|c|}
\hline Group & Mediator & Control & CVI \\
\hline I & IL-15 & 17 & 11 \\
\hline I & IL-1b & 15 & 11 \\
\hline I & IL-4 & 15 & 11 \\
\hline I & MIP-1b & 15 & 8 \\
\hline I & TNFa & 15 & 8 \\
\hline I & GM-CSF & 14 & 6 \\
\hline I & IFNa2 & 14 & 10 \\
\hline I & IL-10 & 14 & 4 \\
\hline I & IL-5 & 14 & 4 \\
\hline I & MIP-1a & 14 & 3 \\
\hline I & IL-1RA & 13 & 6 \\
\hline I & IL-2 & 12 & 7 \\
\hline I & IL-13 & 10 & 3 \\
\hline I & IL-7 & 10 & 6 \\
\hline I & IL-12p40 & 9 & 9 \\
\hline I & IL-12p70 & 8 & 12 \\
\hline I & IL-8 & 8 & 4 \\
\hline I & IL-17A & 7 & 10 \\
\hline I & $\mathrm{IFN} \gamma$ & 4 & 9 \\
\hline I & IL-6 & 4 & 2 \\
\hline I & Eotaxin & 1 & 1 \\
\hline I & MCP-1 & 1 & 1 \\
\hline II & MIG & 1 & 0 \\
\hline II & slL-2ra & 1 & 0 \\
\hline II & IP-10 & 0 & 0 \\
\hline Total & & 118 & 73 \\
\hline
\end{tabular}

CVI, chronic venous insufficiency; GM-CSF, granulocyte macrophage colony stimulating factor.

showing evidence of disrupted expression of inflammatory mediators in CVI patients without VLU (20). We showed that in healthy volunteers (HV), inflammatory mediators exist in the plasma in low levels but are highly connected to one another in a correlation-based network. This may be associated with a homeostatic condition, and to our knowledge, had not previously been studied in this way. In contrast, the blood draining the legs of patients with CVI exhibited not only lower levels of several inflammatory mediators but also a much lower degree of network connectivity, which may reflect a stagnant postinflammatory state (20).

The results of our current study have some important differences. Here, we were able to take blood from competent saphenous veins from control subjects without any sign or symptoms of CVI (CEAP clinical C0). We were also able to compare these values to blood taken from control subjects' arms, to show that the concentration of inflammatory mediators in competent leg veins does not differ from that in the arm. Another important difference between the current study and our previous work is that we were able to amass data from contemporaneously recruited controls. In our previous study, the control samples were banked specimens and were subject to possible storage artifact. Additionally, the number of patient samples was lower in our previous report, and we were able to add analysis of vein samples to the current study.

Here, we demonstrated that patients generally have lower concentrations of many inflammatory mediators when compared to control subjects, as we have noted previously. Strikingly, however, the concentrations of MIG, IP10, and sIL-2r $\alpha$ were significantly higher in refluxing compared to competent leg veins. Elevation of MIG and IP10, as well as sIL2-r $\alpha$, reflect a Th1 response in which IFN $\gamma$-mediated pathways are prominent $(36,37)$. Indeed, IFN $\gamma$ released from Th1 cells induces human venous endothelial cells to secrete MIG and IP10, and can be inhibited by peroxisome proliferator-activated receptor-gamma (PPAR-gamma) agonists (38). In vivo, this could mean that leukocytes may be trapped in the vein walls, promoting release of inflammatory mediators into the local circulation. Such a theory has been posited in the remote past (39-41), but has not been investigated in terms of novel diagnostic or therapeutic tools.

Aside from absolute mediator values, mediator connections were also informative in our data set. Our analysis showed that while MIG, IP10 and sIL2r $\alpha$ were elevated in CVI, they were not connected to other mediators. We have previously suggested this pattern of "elevated but not connected" as a potential marker of pathologic signaling involving IL-6 (14). In some patients who required multiple procedures in a month to treat their refluxing veins, MIG concentrations were relatively lower than those who required only one treatment. Others have found that increasing MIG in wounds is associated with 


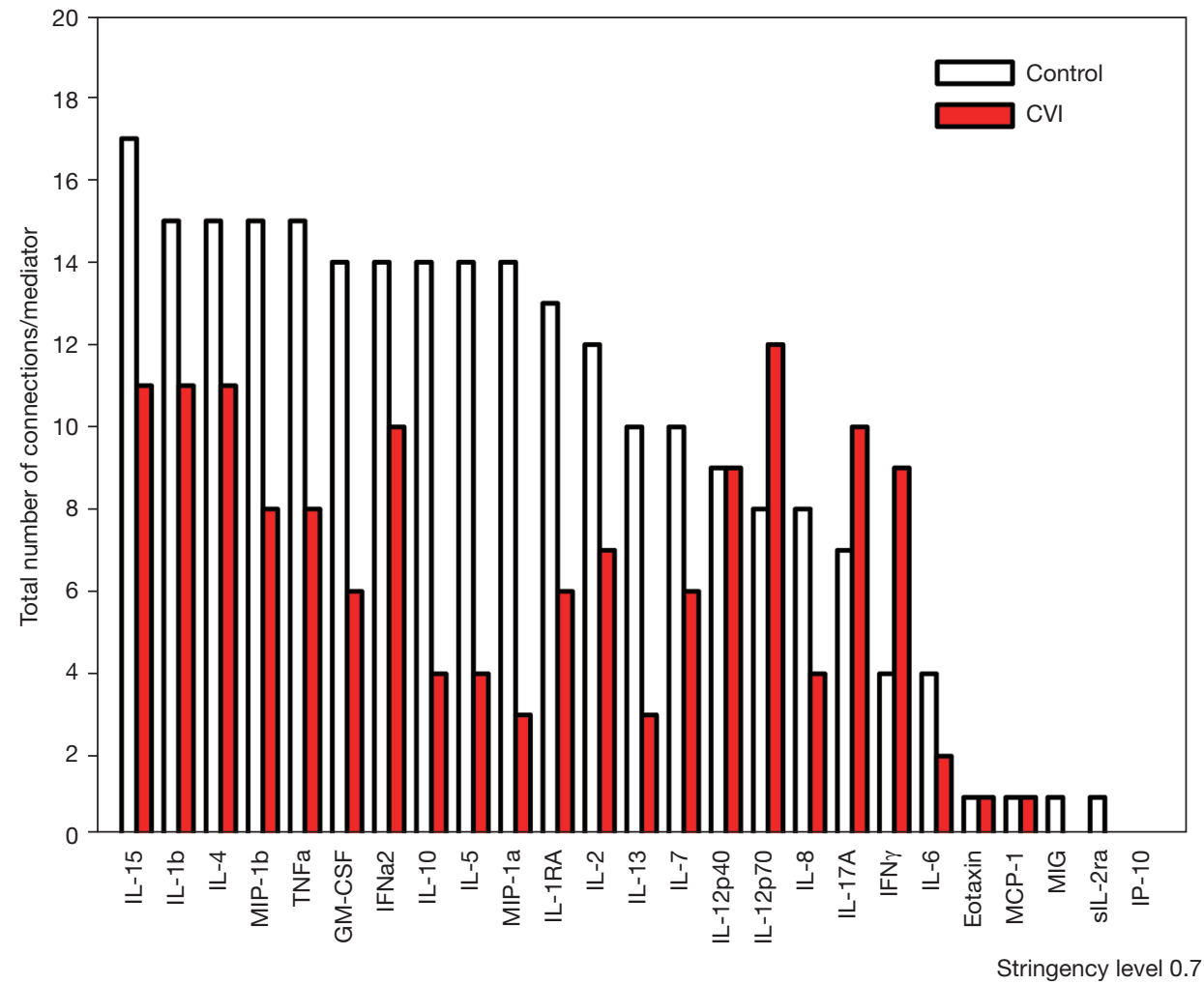

Figure 5 Connections are generally lower in CVI except for few key mediators. The number of correlations for individual mediators are shown for CVI and control subjects $(n=42)$. CVI, chronic venous insufficiency.
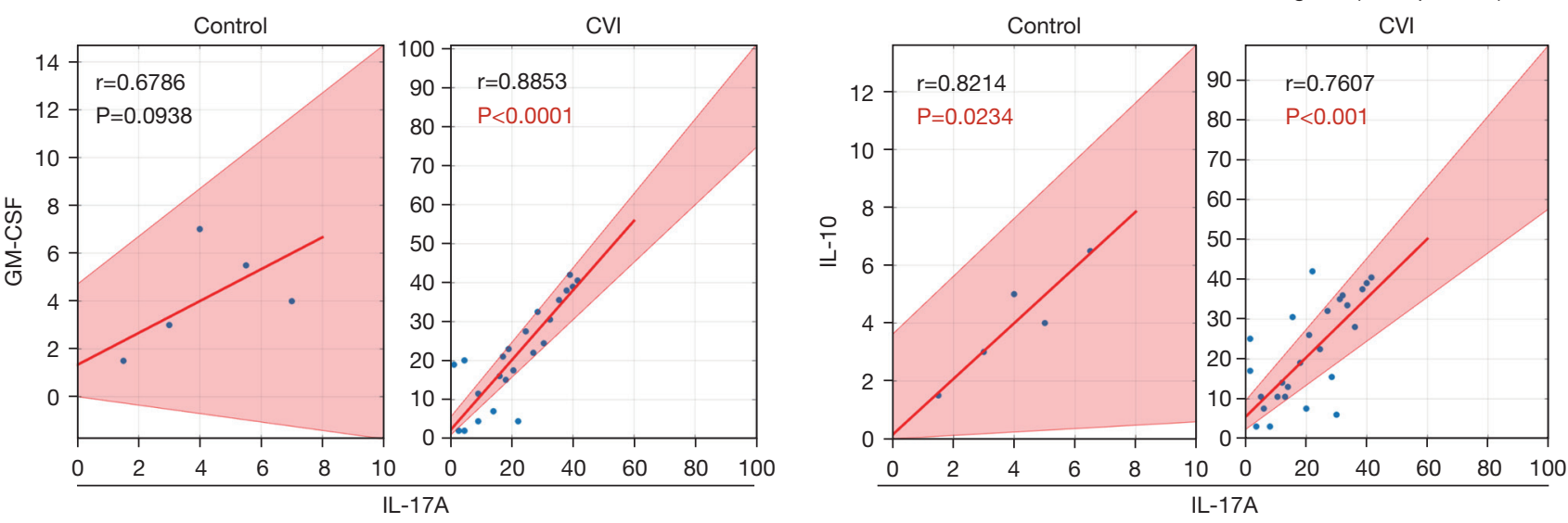

Figure 6 IL-17A correlations suggest involvement of both pathogenic and nonpathogenic Th17. Spearman correlations were performed using MATLAB software to assess relationship between IL-17A and GM-CSF and IL-10 as a reflection of pathogenic and nonpathogenic Th17 cells, respectively $(\mathrm{n}=42)$. Of note, the algorithmic transformation of input data may result in fewer plotted observations. CVI, chronic venous insufficiency; MATLAB, matrix library; GM-CSF, granulocyte macrophage colony stimulating factor; 
healing, whereas stable or decreasing levels correlate with stagnant wounds (42). Our results may follow a similar trajectory.

A pattern also emerged in which some mediators were more connected in CVI than in controls. Those included IFN $\gamma$, IL-17A, IL-12p70 and are reflective of both Th1 and Th17 activity. IL-12p70 is the activated subunit of IL-12 which promotes differentiation of Th1 cells (29). Corroborating with these findings, we found that patterns in Th17 cytokine profiles between the groups. Th17 involvement was suggested in both CVI and control subjects, but a pathogenic Th17 cytokine profile was only seen in CVI.

Our data suggest that Th1 and pathogenic Th17 cells may be present in veins affected by CVI even in the absence of venous ulceration. A role for Th1 responses in mediating chronic structural problems in the cardiovascular system has been posited previously. For example, in patients with cardiac allograft vasculopathy (CAV), chemokine profiling of infiltrating cells in the coronary arteries demonstrated a predominantly Th1 response (43). Similarly, Th1 cells play a critical role in arterial atherosclerosis, another chronic disease involving the vascular system (44). While evidence exists that Th1 and Th17 cells might suppress differentiation of one another (45), others have shown co-existence of both cell types in cardiovascular disease. Specifically, both IFN $\gamma$ (signature cytokine of Th1) and IL17A (signature cytokine of Th17) were found in the same coronary plaques (28).

Our cytokine analyses suggested that an inflammatory process is occurring in VVs. In support of this, we demonstrated that temperature over refluxing veins in patients were higher than those over competent saphenous veins in controls. In either scenario, elevated temperature was seen in patients and correlates with pathology. Temperature may be a useful parameter to assess disease progression with additional study. Applying IRT to skin pathology has been done in other scenarios. Specifically, temperature differences were used successfully to assess resolution of certain parasitic infections involving the skin (21).

There are important limitations in this study. We were not able to obtain blood from the antecubital fossa of patients to compare the two locations. We did not expect the antecubital blood to differ between controls and patients and believed that obtaining blood from the GSV of controls provided a more relevant comparison. Furthermore, we do not have histology of the VVs themselves, since most of our interventions are percutaneous. However, our future efforts will focus on novel imaging techniques to better visualize inflammation in VVs. We did not assess a broad panel of additional inflammatory mediators that may have implicated other inflammatory mechanisms in CVI. Also, patient and control subjects differed strikingly in regards to their BMI, which is thought to increase systemic inflammation, indicated by elevated CRP (46). However, given how specific our sampling was for veins of the legs, we suspect that vein morphology contributes more to our findings than BMI. We are increasing our control database to include patients with a higher BMI but are limited in recruitment due to the COVID-19 pandemic. In addition, we did not sample expression of IL-23, which, akin to IL-12 in Th1 cells, can induce expression of IL-17A from Th17 cells (30). Finally, the correlations that were performed to infer the potential pathogenicity of Th17 cells were based on a low $\mathrm{N}$ for control subjects. This is also a limitation, but the implied results remain intriguing. This is an active area of investigation for our laboratories.

\section{Conclusions}

In conclusion, serum mediators within competent veins show little variability and a high degree of correlation that is generally lacking in plasma obtained from CVI patients within refluxing veins. CVI associates not only with age and BMI, but also with responses consistent with both Th1 and Th17 cell activation. Because VVs are often treated percutaneously, we might be able to use circulating mediators as a reflection of inflammation at the vein wall. Our mediator results, along with temperature elevations, suggest that there may be a local inflammatory process in VVs pathology which contributes to disease progression. Chronic inflammation may mediate damage and should be a target for novel medical therapies, such as those that target Th1 and Th17 cells.

\section{Acknowledgments}

This study was accepted for oral presentation at the American Venous Forum Day of Science, Palm Springs, CA, USA, February 2019.

Funding: This work was supported by the Society for Vascular Surgery Foundation Award (USA).

\section{Footnote}

Reporting Checklist: The authors have completed the 
MDAR reporting checklist. Available at https://dx.doi. org/10.21037/atm-21-688

Data Sharing Statement: Available at https://dx.doi. org/10.21037/atm-21-688

Conflicts of Interest: All authors have completed the ICMJE uniform disclosure form (available at https://dx.doi. org/10.21037/atm-21-688). YV serves as an unpaid editorial board member of Annals of Translational Medicine from March 2019 to February 2021. YV serves on the board of directors of the Society for Complex Acute Illness and is a co-founder of, and stakeholder in Immunetrics Inc., Pittsburgh, PA, USA. Neither of these relationships had any impact on the work presented in this manuscript. US reports this work supported by the SVS Foundation. The other authors have no conflicts of interest to declare.

Ethical Statement: The authors are accountable for all aspects of the work in ensuring that questions related to the accuracy or integrity of any part of the work are appropriately investigated and resolved. The study was conducted in accordance with the Declaration of Helsinki (as revised in 2013). The study was approved by institutional ethics board of the University of Pittsburgh (\#PRO15030072) and informed consent was taken from all individual participants.

Open Access Statement: This is an Open Access article distributed in accordance with the Creative Commons Attribution-NonCommercial-NoDerivs 4.0 International License (CC BY-NC-ND 4.0), which permits the noncommercial replication and distribution of the article with the strict proviso that no changes or edits are made and the original work is properly cited (including links to both the formal publication through the relevant DOI and the license). See: https://creativecommons.org/licenses/by-nc-nd/4.0/.

\section{References}

1. Meissner MH, Gloviczki P, Bergan J, et al. Primary chronic venous disorders. J Vasc Surg 2007;46 Suppl S:54S-67S.

2. Kaplan RM, Criqui MH, Denenberg JO, et al. Quality of life in patients with chronic venous disease: San Diego population study. J Vasc Surg 2003;37:1047-53.

3. Salim S, Machin M, Patterson BO, et al. Global Epidemiology of Chronic Venous Disease: A Systematic
Review with Pooled Prevalence Analysis. Ann Surg 2021;274:971-6.

4. Lal BK. Venous ulcers of the lower extremity: Definition, epidemiology, and economic and social burdens. Semin Vasc Surg 2015;28:3-5.

5. Chaby G, Senet P, Ganry O, et al. Prognostic factors associated with healing of venous leg ulcers: a multicentre, prospective, cohort study. Br J Dermatol 2013;169:1106-13.

6. Peart J. Influence of psychosocial factors on coping and living with a venous leg ulcer. Br J Community Nurs 2015;Suppl Community Wound Care:S21-7.

7. Rich A, McLachlan L. How living with a leg ulcer affects people's daily life: a nurse-led study. J Wound Care 2003;12:51-4.

8. Jones J, Barr W, Robinson J, et al. Depression in patients with chronic venous ulceration. Br J Nurs 2006;15:S17-23.

9. ten Cate-Hoek AJ, Henke PK, Wakefield TW. The post thrombotic syndrome: Ignore it and it will come back to bite you. Blood Rev 2016;30:131-7.

10. Gohel MS, Heatley F, Liu X, et al. A Randomized Trial of Early Endovenous Ablation in Venous Ulceration. N Engl J Med 2018;378:2105-14.

11. Diaz JA, Wrobleski SK, Alvarado CM, et al. P-selectin inhibition therapeutically promotes thrombus resolution and prevents vein wall fibrosis better than enoxaparin and an inhibitor to von Willebrand factor. Arterioscler Thromb Vasc Biol 2015;35:829-37.

12. Culmer DL, Diaz JA, Hawley AE, et al. Circulating and vein wall $\mathrm{P}$-selectin promote venous thrombogenesis during aging in a rodent model. Thromb Res 2013;131:42-8.

13. Cushman M, Callas PW, Allison MA, et al. Inflammation and peripheral venous disease. The San Diego Population Study. Thromb Haemost 2014;112:566-72.

14. Mi Q, Constantine G, Ziraldo C, et al. A dynamic view of trauma/hemorrhage-induced inflammation in mice: principal drivers and networks. PLoS One 2011;6:e19424.

15. Namas RA, Vodovotz Y, Almahmoud K, et al. Temporal Patterns of Circulating Inflammation Biomarker Networks Differentiate Susceptibility to Nosocomial Infection Following Blunt Trauma in Humans. Ann Surg 2016;263:191-8.

16. Zamora R, Vodovotz Y, Mi Q, et al. Data-Driven Modeling for Precision Medicine in Pediatric Acute Liver Failure. Mol Med 2017;22:821-9.

17. Abboud A, Namas RA, Ramadan M, et al. Computational Analysis Supports an Early, Type 17 Cell-Associated Divergence of Blunt Trauma Survival and Mortality. Crit 
Care Med 2016;44:e1074-81.

18. Azhar N, Ziraldo C, Barclay D, et al. Analysis of serum inflammatory mediators identifies unique dynamic networks associated with death and spontaneous survival in pediatric acute liver failure. PLoS One 2013;8:e78202.

19. Tohme S, Yazdani HO, Sud V, et al. Computational Analysis Supports IL-17A as a Central Driver of Neutrophil Extracellular Trap-Mediated Injury in Liver Ischemia Reperfusion. J Immunol 2019;202:268-77.

20. Sachdev U, Vodovotz L, Bitner J, et al. Suppressed networks of inflammatory mediators characterize chronic venous insufficiency. J Vasc Surg Venous Lymphat Disord 2018;6:358-66.

21. Schuster A, Thielecke M, Raharimanga V, et al. Highresolution infrared thermography: a new tool to assess tungiasis-associated inflammation of the skin. Trop Med Health 2017;45:23.

22. Lurie F, Passman M, Meisner M, et al. The 2020 update of the CEAP classification system and reporting standards. J Vasc Surg Venous Lymphat Disord 2020;8:34252. Erratum in: J Vasc Surg Venous Lymphat Disord 2021;9:288.

23. Almeida JI, Wakefield T, Kabnick LS, et al. Use of the Clinical, Etiologic, Anatomic, and Pathophysiologic classification and Venous Clinical Severity Score to establish a treatment plan for chronic venous disorders. J Vasc Surg Venous Lymphat Disord 2015;3:456-60.

24. Solovyev A, Mi Q, Tzen YT, et al. Hybrid equation/agentbased model of ischemia-induced hyperemia and pressure ulcer formation predicts greater propensity to ulcerate in subjects with spinal cord injury. PLoS Comput Biol 2013;9:e1003070.

25. Ziraldo C, Mi Q, An G, et al. Computational Modeling of Inflammation and Wound Healing. Adv Wound Care (New Rochelle) 2013;2:527-37.

26. Pernet CR, Wilcox R, Rousselet GA. Robust correlation analyses: false positive and power validation using a new open source matlab toolbox. Front Psychol 2013;3:606.

27. Bergamaschi C, Pandit H, Nagy BA, et al. Heterodimeric IL-15 delays tumor growth and promotes intratumoral CTL and dendritic cell accumulation by a cytokine network involving XCL1, IFN- $\gamma$, CXCL9 and CXCL10. J Immunother Cancer 2020;8:e000599.

28. Eid RE, Rao DA, Zhou J, et al. Interleukin-17 and interferon-gamma are produced concomitantly by human coronary artery-infiltrating $\mathrm{T}$ cells and act synergistically on vascular smooth muscle cells. Circulation 2009; 119:1424-32.
29. Zhang S, Wang Q. Factors determining the formation and release of bioactive IL-12: regulatory mechanisms for IL-12p70 synthesis and inhibition. Biochem Biophys Res Commun 2008;372:509-12.

30. Moschen AR, Tilg H, Raine T. IL-12, IL-23 and IL-17 in IBD: immunobiology and therapeutic targeting. Nat Rev Gastroenterol Hepatol 2019;16:185-96.

31. Kumar P, Subramaniyam G. Molecular underpinnings of Th17 immune-regulation and their implications in autoimmune diabetes. Cytokine 2015;71:366-76.

32. Lee $\mathrm{Y}$, Awasthi A, Yosef N, et al. Induction and molecular signature of pathogenic TH17 cells. Nat Immunol 2012;13:991-9.

33. Nathan C. Points of control in inflammation. Nature 2002;420:846-52.

34. Okin D, Medzhitov R. Evolution of inflammatory diseases. Curr Biol 2012;22:R733-40.

35. Naoum JJ, Hunter GC. Pathogenesis of varicose veins and implications for clinical management. Vascular 2007;15:242-9.

36. Berthoud TK, Dunachie SJ, Todryk S, et al. MIG (CXCL9) is a more sensitive measure than IFN-gamma of vaccine induced $\mathrm{T}$-cell responses in volunteers receiving investigated malaria vaccines. J Immunol Methods 2009;340:33-41. Erratum in: J Immunol Methods 2011;368:84.

37. Lauw FN, Simpson AJ, Prins JM, et al. The CXC chemokines gamma interferon (IFN-gamma)-inducible protein 10 and monokine induced by IFN-gamma are released during severe melioidosis. Infect Immun 2000;68:3888-93.

38. Marx N, Mach F, Sauty A, et al. Peroxisome proliferatoractivated receptor-gamma activators inhibit IFN-gammainduced expression of the $\mathrm{T}$ cell-active CXC chemokines IP-10, Mig, and I-TAC in human endothelial cells. J Immunol 2000;164:6503-8.

39. Saharay M, Shields DA, Porter JB, et al. Leukocyte activity in the microcirculation of the leg in patients with chronic venous disease. J Vasc Surg 1997;26:265-73.

40. Thomas PR, Dormandy JA. White cell and platelet trapping in patients with chronic venous insufficiency. Phlebologie 1988;41:771-6.

41. Thomas PR, Nash GB, Dormandy JA. White cell accumulation in dependent legs of patients with venous hypertension: a possible mechanism for trophic changes in the skin. Br Med J (Clin Res Ed) 1988;296:1693-5.

42. Edsberg LE, Wyffels JT, Brogan MS, et al. Analysis of the proteomic profile of chronic pressure ulcers. Wound 
Page 14 of 14

Repair Regen 2012;20:378-401.

43. van Loosdregt J, van Oosterhout MF, Bruggink AH, et al. The chemokine and chemokine receptor profile of infiltrating cells in the wall of arteries with cardiac allograft vasculopathy is indicative of a memory T-helper 1 response. Circulation 2006;114:1599-1607.

44. van Duijn J, Kritikou E, Benne N, et al. CD8+

T-cells contribute to lesion stabilization in advanced

Cite this article as: Vodovotz L, Zamora R, Barclay DA, Vodovotz Y, Yin J, Bitner J, Florida J, Avgerinos ED, Sachdev U. Inflammatory signals and network connections implicate cellmediated immunity in chronic venous insufficiency. Ann Transl Med 2021;9(22):1643. doi: 10.21037/atm-21-688

\section{Vodovotz et al. Inflammatory signals in venous insufficiency}

atherosclerosis by limiting macrophage content and CD4+ T-cell responses. Cardiovasc Res 2019;115:729-38.

45. Lin H, Tong ZH, Xu QQ, et al. Interplay of Th1 and Th17 cells in murine models of malignant pleural effusion. Am J Respir Crit Care Med 2014;189:697-706.

46. Visser M, Bouter LM, McQuillan GM, et al. Elevated C-reactive protein levels in overweight and obese adults. JAMA 1999;282:2131-5. 